\title{
Analytical Delay Models for VLSI Interconnects Under Ramp Input
}

\author{
Andrew B. Kahng, Kei Masuko, and Sudhakar Muddu* \\ UCLA Computer Science Department, Los Angeles, CA 90095-1596 USA \\ Cadence Design Systems, Inc., San Jose CA 95134 \\ Silicon Graphics, Inc., Mountain View, CA 94039 \\ abk@cs.ucla.edu,masuko@cs.ucla.edu,sudhakar@cs.ucla.edu
}

\begin{abstract}
Elmore delay has been widely used as an analytical estimate of interconnect delays in the performance-driven synthesis and layout of VLSI routing topologies. However, for typical $R L C$ interconnections with ramp input, Elmore delay can deviate by up to $100 \%$ or more from SPICEcomputed delay since it is independent of rise time of the input ramp signal. We develop new analytical delay models based on the first and second moments of the interconnect transfer function when the input is a ramp signal with finite rise time. Delay estimates using our first moment based analytical models are within $4 \%$ of SPICE-computed delay, and models based on both first and second moments are within $2.3 \%$ of SPICE, across a wide range of interconnect parameter values. Evaluation of our analytical models is several orders of magnitude faster than simulation using SPICE. We also describe extensions of our approach for estimation of source-sink delays in arbitrary interconnect trees.
\end{abstract}

\section{Introduction}

Accurate calculation of propagation delay in VLSI interconnects is critical to the design of high speed systems, and transmission line effects now play an important role in determining interconnect delays and system performance. Existing techniques are based on either simulation or (closed-form) analytical formulas. Simulation methods such as SPICE give the most accurate insight into arbitrary interconnect structures, but are computationally expensive. Faster methods based on moment match ing techniques are proposed in $[12,13,14,17]$, but are still too expensive to be used during layout optimization. Thus, Elmore delay [2], a first order approximation of delay under step input, is still the most widely used delay model in the performance-driven synthesis of clock distribution and Steiner global routing topologies. However, Elmore delay cannot be applied to estimate the delay for interconnect lines with ramp input source; this inaccuracy is harmful to current performance-driven routing methods which try to determine optimal interconnect segment lengths and widths (as well as driver sizes). Previous moment-based approaches $[12,14,17]$ can compute a response for interconnects under ramp input within a simulation-based methodology, but no previous work has given analytical delay estimation models based on the first few moments.

Recently, [3] presented lower and upper bounds for the ramp input response; their delay model is the same as the Elmore model for ramp input (we refer to this model as analytical ramp input model $\left(T_{A D}\right)$ in this paper). Delay estimates for the analytical ramp input model are off by as much as $50 \%$ from SPICE-computed delays for $50 \%$ threshold voltage, and the analytical ramp input model cannot be used to obtain threshold delay for various threshold voltages. The authors of [5] used Elmore delay as an upper bound on the 50\% threshold delay for $R C$ interconnection lines under arbitrary input waveforms. However, we find that Elmore delay is not at all close to SPICE-computed 50\% threshold delay and, depending on the input slew time and driver resistance, can be either greater or less than SPICE-computed delay (see Section 7 below). This paper gives a new and accurate analytical delay estimate for distributed $R L C$ interconnects under ramp input. To experimentally validate our analysis and delay formula, we model VLSI interconnect lines having various combinations of source, and load parameters, ap-

${ }^{*}$ Principal author, to whom correspondence should be addressed: Sudhakar Muddu (muddu@mti.sgi.com). Andrew B. Kahng is currently on Sabbatical leave at Cadence Design Systems, ak@cadence.com. ply different input rise times, and obtain delay estimates from SPICE, Elmore delay and the proposed analytical delay model. Over our range of test cases, Elmore delay estimates can vary by as much as $100 \%$ from SPICE-computed delays. As the input rise time increases, Elmore delay deviates even further from SPICE-computed delays. In contrast, our single-pole delay estimates are within $4 \%$ of SPICE delays and our twopole delay estimates are within $2.3 \%$ of SPICE delays. ${ }^{1}$ Since our analytical models have the same time complexity of evaluation as the Elmore model, we believe that they are very useful for performance-driven routing methodologies.

The organization of our paper is as follows. In Section 2 we discuss delay models which have been previously proposed for interconnect lines under step input. Section 3 presents a new analytical delay definition for interconnect lines under ramp input. Section 4 discusses various threshold delay models for single-pole approximation of the interconnect transfer function; Section 5 gives various threshold delay models for two-pole approximation; and Section 6 extends our delay modeling approach to interconnection trees. Section 7 concludes with experimental results for various combinations of input rise times and interconnect parameters.

\section{Previous Delay Models Under Step Input}

The transfer function of an $R L C$ interconnect line with source and load impedance (Figure 1) can be obtained using ABCD parameters [1] as

$$
\begin{aligned}
H(s) & =\frac{1}{\left[\cosh (\theta h)+\frac{Z_{S}}{Z_{0}} \sinh (\theta h)\right]+\frac{1}{Z_{T}}\left[Z_{0} \sinh (\theta h)+Z_{S} \cosh (\theta h)\right]} \\
& =\frac{1}{1+b_{1} s+b_{2} s^{2}+\ldots+b_{k} s^{k}+\ldots}
\end{aligned}
$$

where $\theta=\sqrt{(r+s l) s c}$ is the propagation constant and $Z_{0}=\sqrt{\frac{R+s L}{s C}}$ is the characteristic impedance; $r=\frac{R}{h}, l=\frac{L}{h}, c=\frac{C}{h}$ are resistance, inductance, and capacitance per unit length and $h$ is the length of the line. The variables $b_{k}$ are called the coefficients of the transfer function and are directly related to the moments of the transfer function [8]. Expanding the transfer function into a Maclaurin series of $s$ around $s=0$ leads to an infinite series, and to compute the response the series is truncated to desired order. The method of Padé approximation has been widely used to compute the response from the transfer function [11, 12]. For the case of resistive source $\left(R_{S}\right)$ and capacitive load $\left(C_{L}\right)$ impedances, the coefficient of $s$ in the transfer function can be obtained as [8] $b_{1}=$ $R_{S} C+R_{S} C_{L}+\frac{R C}{2}+R C_{L}$.

Efficient delay estimates for interconnect lines are typically derived by considering a single interconnect line with resistive source and capacitive load impedances; delay formulas for an interconnect tree come from recursive application of the formula for a single line. Elmore delay [2] is a first order delay estimate for interconnect lines under step input. It is equal to the first moment of the system impulse response, i.e., the coefficient of $s$ or the first moment in the system transfer function $H(s)$. Applying this definition to $H(s)$ in Equation (1), we see that the Elmore delay is equal to the coefficient $b_{1}$.

\footnotetext{
${ }^{1}$ We use threshold delay to refer to delay measured from the point when the input signal is zero. To compute delay relative to the input signal, subtract the corresponding threshold delay of the input signal (e.g., for $50 \%$ threshold voltage, the delay for the input ramp is $\frac{T_{R}}{2}$ ).
} 


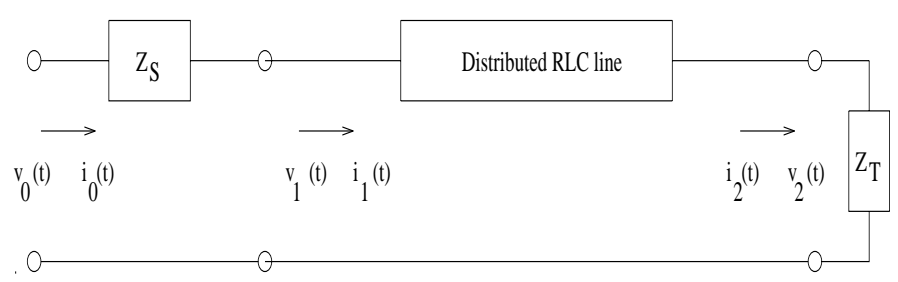

Figure 1: 2-port model of a distributed $R L C$ line with source impedance $Z_{S}$ and load impedance $Z_{T}$.

By considering only one pole in the transfer function, i.e, approximating the denominator polynomial to only the first moment, the single pole response can be obtained as in $[4,15]$. The single pole of the transfer function is equal to the inverse of the Elmore delay $T_{E D}$. Hence, the delay at arbitrary thresholds of the single pole response can be directly related to Elmore delay (Elmore delay actually corresponds to the $63.2 \%$ threshold voltage of the single pole response). For example, delay at $50 \%$ threshold voltage is $0.69 b_{1}$, and delay at $90 \%$ threshold voltage is $2.3 b_{1}$. Although Elmore delay has been widely used for interconnect timing analysis, it cannot accurately estimate the delay for $R L C$ interconnect lines, which are the appropriate representation for interconnects whose inductive impedance cannot be neglected [6]. ${ }^{2}$ More critically, Elmore delay cannot estimate delays when the input signal is a ramp.

\section{Analytical Ramp Delay Definitions}

In practice, the input at any gate or root of a tree is a ramp with finite rise (or fall) time, and there are no published analytical delay models for ramp input. We now propose various ramp delay definitions and also compute analytical delay expressions using the first one or two moments of the transfer function. We discuss delay models for rising ramp input only, since our analyses can be easily extended for falling ramp input [9].

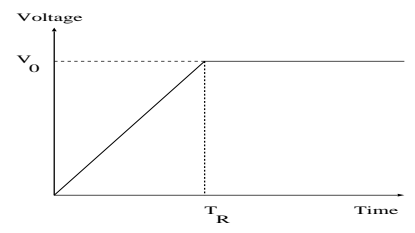

(a)

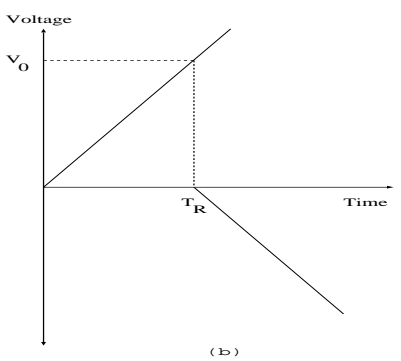

Figure 2: A ramp input function: (a) finite ramp with rise time $T_{R}$, and (b) finite ramp decomposed into two shifted infinite ramps.

\section{Rising Ramp Input}

The finite rising ramp input shown in Figure 2 can be expressed in the time domain as

$$
v_{\text {in }}(t)=\frac{V_{0}}{T_{R}}\left[t U(t)-\left(t-T_{R}\right) U\left(t-T_{R}\right)\right] \quad \text { for all } t \geq 0
$$

where $U(t)$ denotes the step function. The finite ramp input in the transform domain is $V_{i n}(s)=\frac{V_{0}}{T_{R}} \cdot \frac{1}{s^{2}}\left[1-e^{-s T_{R}}\right]$. In the transform domain, the output response is

$$
V_{\text {out }}(s)=V_{\text {in }}(s) H(s)=\frac{V_{0}}{T_{R}} \cdot \frac{1}{s^{2}}\left[1-e^{-s T_{R}}\right] H(s) .
$$

\footnotetext{
${ }^{2}$ Recently, [8] have developed a more accurate analytical delay model considering inductive effects based on the first and second moments of the transfer function. Their model gives accurate estimates compared to SPICE-computed delays, but is valid only for step inputs.
}

We now give two distinct derivations of an analytical ramp delay estimate.

\section{Elmore Definition.}

Applying Elmore's original definition of delay for step input [2] yields an analytical delay $T_{A D}$ for ramp input, i.e.,

$$
T_{A D}=\frac{1}{V_{0}} \int_{0}^{\infty} t v_{\text {out }}^{\prime}(t) d t
$$

where $v_{\text {out }}^{\prime}(t)$ is the derivative of the output response under finite ramp input. Taking the Laplace transform of $v_{\text {out }}^{\prime}(t)$,

$$
V_{\text {out }}^{\prime}(s)=\int_{0}^{\infty} v_{\text {out }}^{\prime}(t) d t-s \int_{0}^{\infty} t v_{\text {out }}^{\prime}(t) d t+\ldots
$$

Equation (2) then implies that the analytical ramp input delay $T_{A D}$ in the time domain is equal to the first moment of the derivative of the response. In the transform domain, $T_{A D}$ is equal to the first moment (or coefficient of $s$ ) of the function $\frac{V_{\text {out }}^{\prime}(s)}{V_{0}}$, which is equal to $s \cdot \frac{V_{\text {out }}(s)}{V_{0}}$. The derivative of the response in the transform domain is

$$
V_{\text {out }}^{\prime}(s)=V_{0}\left(1-\frac{s T_{R}}{2}+\ldots\right) \frac{1+a_{1} s+a_{2} s^{2}+\ldots}{1+b_{1} s+b_{2} s^{2}+\ldots}
$$

Therefore, the analytical ramp input delay is

$$
T_{A D}=\frac{T_{R}}{2}+b_{1}-a_{1}=\frac{T_{R}}{2}+T_{E D}
$$

where $T_{E D}$ is the Elmore delay for a step input (i.e., the first moment of the transfer function). Another definition of delay based on the formula given in [10] yields the same result of Equation (3) [9].

\section{Group Delay Definition.}

The concept of group delay was initially defined for step input by Vlach et al. [18]. We now give a group delay definition for computing ramp input delay similar to that in [18], and show that it converges to the same analytical expression of Equation (3).

Recall that group delay is defined as the negative of the rate of change of the phase characteristic $\phi$ of the output response $V_{\text {out }}(\omega)$ with respect to frequency, at zero frequency, i.e., $T_{G D}=\lim _{\omega \rightarrow 0}-\frac{\partial \phi}{\partial \omega}$. To compute the phase characteristic of the output response, we first compute the output response $V_{\text {out }}(s)$ in the transform domain and then substitute for the Laplace variable $s=j \omega$, i.e.,

$$
\begin{aligned}
V_{\text {out }}(\omega) & =\frac{V_{0}}{T_{R}} \cdot \frac{-1}{\omega^{2}}\left(1-e^{-j \omega T_{R}}\right) \cdot H(\omega) \\
& =\frac{-V_{0}}{T_{R} \omega}\left[\left(\frac{\omega T_{R}^{2}}{2}-\frac{\omega^{3} T_{R}^{4}}{3 !}+\ldots\right)+j\left(T_{R}-\frac{\omega^{2} T_{R}^{3}}{3 !}+\ldots\right)\right] H(\omega) \\
& =\frac{-V_{0}}{T_{R} \omega}\left[M_{1}+j M_{2}\right] H(\omega)
\end{aligned}
$$

where $M_{1}$ and $M_{2}$ are the real and imaginary parts of the input ramp function. Writing the transfer function in terms of numerator and denominator polynomials,

$$
H(\omega)=\frac{\left(1-a_{2} \omega^{2}+\ldots\right)+j\left(a_{1} \omega-a_{3} \omega^{3}+\ldots\right)}{\left(1-b_{2} \omega^{2}+\ldots\right)+j\left(b_{1} \omega-b_{3} \omega^{3}+\ldots\right)}=\frac{N_{1}+j N_{2}}{D_{1}+j D_{2}}
$$

Then, the phase characteristic of the output response is $\phi=\tan ^{-1} \frac{M_{2}}{M_{1}}+$ $\tan ^{-1} \frac{N_{2}}{N_{1}}-\tan ^{-1} \frac{D_{2}}{D_{1}}$. We obtain the group delay as

$$
T_{G D}=\lim _{\omega \rightarrow 0}-\frac{\partial \phi}{\partial \omega}=\frac{T_{R}}{2}+b_{1}-a_{1}
$$




\section{Single-Pole Analysis}

If we approximate the system transfer function up to the first moment (or coefficient of $s), H(s) \approx \frac{1}{1+s b_{1}}$. Then, the output response under infinite ramp is ${ }^{3}$

$$
U_{\text {out }}(s)=\frac{V_{0}}{T_{R}} \frac{1}{s^{2}} \frac{1}{1+s b_{1}}=\frac{V_{0}}{T_{R}}\left[\frac{1}{s^{2}}-\frac{b_{1}}{s}+\frac{b_{1}}{\left(s+1 / b_{1}\right)}\right]
$$

with corresponding time-domain response

$$
u_{\text {out }}(t)=\frac{V_{0}}{T_{R}}\left[-b_{1}+t+b_{1} e^{\frac{-t}{b_{1}}}\right]
$$

The time-domain response for a finite ramp is therefore

$$
\begin{aligned}
v_{\text {out }}(t) & =u_{\text {out }}(t)-u_{\text {out }}\left(t-T_{R}\right) \\
& =\frac{V_{0}}{T_{R}}\left[T_{R}+b_{1} e^{\frac{-t}{b_{1}}}-b_{1} e^{\frac{-\left(t-T_{R}\right)}{b_{1}}}\right]
\end{aligned}
$$

Note that as $t \rightarrow \infty, v_{\text {out }}(t)$ tends to a final value of $V_{0}$ as expected. [16] used a similar single-pole analysis to compute delay and transition times solving the above response equations by applying Newton-Raphson iteration.

\subsection{Analytical Delay Model}

It turns out that using the analytical ramp delay computed using the definition in Section 3 and the output response given in Equations (4) and (5) leads to the same result:

$$
\begin{aligned}
T_{A D} & =\frac{1}{V_{0}} \int_{0}^{T_{R}} t u_{\text {out }}^{\prime}(t) d t+\frac{1}{V_{0}} \int_{T_{R}}^{\infty} t v_{\text {out }}^{\prime}(t) d t \\
& =\frac{T_{R}}{2}+b_{1}
\end{aligned}
$$

\section{Threshold Voltage Corresponding To Analytical Ramp Delay.}

Section 3 gave two different methods for computing an analytical ramp input delay from the output response. The threshold voltage corresponding to this analytical delay is not known, and must be computed by substituting $T_{A D}$ for time in either the infinite or the finite ramp responses.

Computing the threshold voltage for the infinite ramp response in Equation (4) for $b_{1} \ll \frac{T_{R}}{2}$, we get

$$
u_{\text {out }}\left(t=T_{A D}\right)=\frac{V_{0}}{2}\left[1+\frac{2 b_{1}}{T_{R}} e^{-\left(1+\frac{1}{2 b_{1} / T_{R}}\right)}\right] .
$$

In the limit as $\frac{2 b_{1}}{T_{R}} \rightarrow 0$ the threshold voltage reduces to $u_{\text {out }}\left(t=T_{A D}\right)=$ $\frac{V_{0}}{2}$. Hence, for large rise-times or small first moment of the transfer function the analytical delay $T_{A D}$ corresponds to $50 \%$ threshold voltage. When $b_{1} \gg \frac{T_{R}}{2}$, using the finite ramp response in Equation (5) gives

$$
v_{\text {out }}\left(t=T_{A D}\right)=V_{0}\left[1+\frac{1}{2 e} \frac{2 b_{1}}{T_{R}}\left(e^{\frac{-1}{2 b_{1} / T_{R}}}-e^{\frac{1}{2 b_{1} / T_{R}}}\right)\right] .
$$

In the limit as $\frac{2 b_{1}}{T_{R}} \rightarrow \infty$ the threshold voltage reduces to $v_{\text {out }}\left(t=T_{A D}\right)=$ $V_{0}(1-1 / e)=0.632 V_{0}$. Hence, for small rise-times or large first moment of the transfer function the analytical delay $T_{A D}$ corresponds to $63.2 \%$ threshold voltage. We see that for any choice of $T_{R}$ and $b_{1}$ the threshold voltage corresponding to the analytical delay $T_{A D}$ will be between $50 \%$ and $63.2 \%$.

\footnotetext{
${ }^{3}$ In the transform and time domains, we respectively use $U(x, s)$ and $u(x, t)$ to indicate the response for infinite ramp input, and $V(x, s)$ and $v(x, t)$ to indicate the response for finite ramp input.
}

\subsection{Threshold Delay Models}

\section{Condition for Computing Threshold Delay Using Finite or Infinite Ramp Response.}

The ramp input delay at any threshold voltage can be computed using the infinite ramp response in Equation (4) if the ramp delay is less than the rise time $T_{R}$, or using the finite ramp response in Equation (5) if the ramp delay is greater than $T_{R}$. For example, the delay at threshold Th1 in Figure 3 is computed using the infinite ramp response, and the delay at threshold $T h 2$ is computed using the finite ramp response. To determine when the infinite ramp response should be used, we write the threshold voltage corresponding to the rise-time $T_{R}$ in terms of interconnect and rise time parameters:

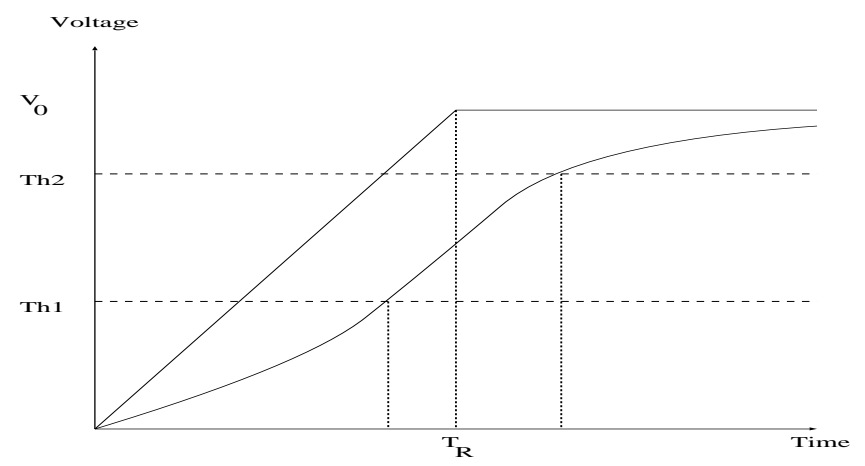

Figure 3: Ramp input delay at various threshold voltages.

$$
v_{T_{R}}=\left[1-\frac{b_{1}}{T_{R}}\left(1-e^{\frac{-1}{b_{1} / T_{R}}}\right)\right]
$$

Here, $v_{T_{R}}$ is the threshold voltage at which the delay through the interconnect is equal to $T_{R}$. Let $v_{t h}$ be the threshold voltage of interest for the finite ramp response, expressed as a fraction of the steady state voltage $V_{0}$. If $v_{t h} \leq v_{T_{R}}$, delay is calculated using Equation (4), and if $v_{t h}>v_{T_{R}}$, delay is calculated using Equation (5).

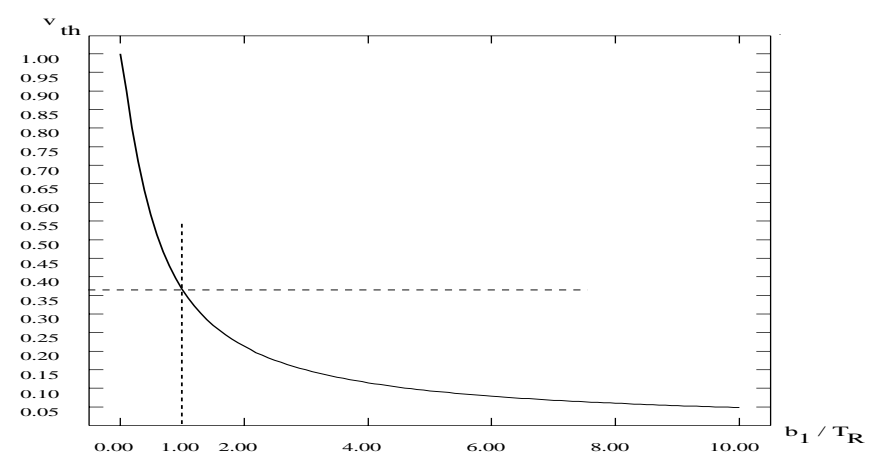

Figure 4: Variation of threshold voltage at delay equal to rise-time $T_{R}$ with respect to the factor $\frac{b_{1}}{T_{R}}$.

Observe that Equation (6) can be rearranged to obtain a condition on $\frac{b_{1}}{T_{R}}$ for any given threshold voltage $v_{t h}$ : the condition for delay calculation using infinite ramp response is

$$
\frac{b_{1}}{T_{R}}\left(1-e^{\frac{-1}{b_{1} / T_{R}}}\right) \leq\left(1-v_{t h}\right)
$$

and the condition for delay calculation using finite ramp response is

$$
\frac{b_{1}}{T_{R}}\left(1-e^{\frac{-1}{b_{1} / T_{R}}}\right) \geq\left(1-v_{t h}\right)
$$


Figure 4 shows the variation of $v_{T_{R}}$ with respect to the factor $\frac{b_{1}}{T_{R}}$. At $b_{1}=T_{R}$ the threshold voltage $v_{T_{R}}$ is $0.368 V_{0}$, i.e., $36.8 \%$. Since most sub-micron interconnect networks have small rise-times and large propagation delays, the delays at threshold voltages of interest $(50 \%$ or $90 \%)$ will likely be computed by considering the finite ramp response as developed in Equation (9) below. 4

\section{Threshold Delay Using Infinite Ramp Response.}

Model 1. For the infinite ramp response of Equation (4), the threshold delay is

$$
T_{R D 1}+b_{1} e^{\frac{-T_{R D 1}}{b_{1}}}=u_{t h} T_{R}+b_{1}
$$

where $u_{t h}$ is the threshold voltage of interest for the infinite ramp response. We can solve such a recursive equation in less than 10 iterations of simple back-substitution (with $T_{A D}$ as the starting value) for all the interconnect configurations we considered. To obtain a closed-form delay formula, we approximate $T_{R D 1}$ in the exponential term with some $f\left(T_{A D}\right)$, which yields

$$
T_{R D 1}=u_{t h} T_{R}+b_{1}\left(1-e^{\frac{-f\left(T_{A D}\right)}{b_{1}}}\right) .
$$

Here, $f\left(T_{A D}\right)$ depends on the threshold voltage and $T_{A D} .{ }^{5}$ The above delay estimate can be improved by expressing $T_{R D 1}$ as $u_{t h} T_{R}+\tau_{R D 1}$, since the threshold delay for the infinite ramp response $T_{R D 1}$ is greater than the threshold delay for the infinite ramp input $u_{t h} T_{R}$. Making this change in delay variable in Equation (4), we get

$$
-b_{1}+\tau_{R D 1}+b_{1} e^{-\frac{u_{t h} T_{R}+\tau_{R D 1}}{b_{1}}}=0
$$

Expanding $e^{-\frac{\tau_{R D 1}}{b_{1}}}$ as a Taylor series and considering only the first three terms yields

$$
\tau_{R D 1}^{2}+2 b_{1}\left(e^{\frac{u_{t h} T_{R}}{b 1}}-1\right) \tau_{R D 1}-2 b_{1}^{2}\left(e^{\frac{u_{b} T_{R}}{b 1}}-1\right)=0
$$

Solving for $\tau_{R D 1}$ in the above equation, the threshold delay can be expressed as

$$
T_{R D 1}=u_{t h} T_{R}+b_{1}\left(1-e^{\frac{u_{h} T_{R}}{b 1}}+\sqrt{e^{\frac{2 u_{h} T_{R}}{b 1}}-1}\right)
$$

Using this $T_{R D 1}$ value for $f\left(T_{A D}\right)$ in the exponential term of the Equation (7), we obtain delay values that are very close to the values obtained by solving the equation through iteration.

\section{Threshold Delay Using Finite Ramp Response.}

Model 2. For the finite ramp response of Equation (5),

$$
v_{t h}=\frac{1}{T_{R}}\left[T_{R}+b_{1} e^{\frac{-T_{R D 2}}{b_{1}}}-b_{1} e^{\frac{-\left(T_{R D 2}-T_{R}\right)}{b_{1}}}\right] \text {. }
$$

Collecting the threshold delay $T_{R D 2}$ terms, we obtain

$$
T_{R D 2}=b_{1}\left|\ln \left(\frac{b_{1}}{T_{R}} \cdot \frac{\left(e^{\frac{1}{b_{1} / T_{R}}}-1\right)}{\left(1-v_{t h}\right)}\right)\right|=b_{1}\left|\ln \left(\frac{F_{1}}{\left(1-v_{t h}\right)}\right)\right|
$$

${ }^{4}$ At $50 \%$ threshold, the condition for delay calculation using infinite ramp response is $\frac{b_{1}}{T_{R}} \leq 0.625$, with delay calculated using finite ramp response otherwise. Similarly, at $90 \%$ threshold, the condition for delay calculation using infinite ramp response is $\frac{b_{1}}{T_{R}} \leq 0.1$.

${ }^{5}$ In [9] the function $f\left(T_{A D}\right)$ is approximated by $f\left(T_{A D}\right)=T_{A D} \ln \left(\frac{1}{1-u_{t h}}\right)$, which is threshold delay for the system with analytical delay as the time constant. The delay estimates using this approximation are reasonably close to SPICE-computed delays. where the factor $F_{1}=\frac{b_{1}}{T_{R}}\left(e^{\frac{1}{b_{1} / T_{R}}}-1\right)$ can vary between $\infty$ and 0 . With such a large variation in $F_{1}$, it is very difficult to fit the threshold delay $T_{R D 2}$ against the corresponding SPICE delay.

Model 3. Since the threshold delay computed from the finite ramp response is greater than $T_{R}$, an alternative formula for the threshold delay can be obtained by expressing $T_{R D 3}$ as $T_{R D 3}=T_{R}+\tau_{R D 3}$. Substituting into Equation (5) yields

$$
v_{t h}=\frac{1}{T_{R}}\left[T_{R}+b_{1} e^{\frac{-T_{R}}{b_{1}}} e^{\frac{-\tau_{R D 3}}{b_{1}}}-b_{1} e^{\frac{-\tau_{R D 3}}{b_{1}}}\right]
$$

Therefore, the delay is

$$
T_{R D 3}=T_{R}+b_{1}\left|\ln \left(\frac{F_{2}}{\left(1-v_{t h}\right)}\right)\right|
$$

The factor $F_{2}=\frac{b_{1}}{T_{R}}\left(1-e^{\frac{-1}{b_{1} / T_{R}}}\right)$ varies between 0 and 1.0 as shown in Figure 5. For $b_{1}=T_{R}$ this factor is $F_{2}=0.632$. For $b_{1}>T_{R}$ we can find a good approximation for $F_{2}$ by fitting against SPICE-computed delays, since the variation in $F_{2}$ values is very small. However, for the range of interconnect configurations studied both Model 2 and Model 3 gave essentially identical results and hence Section 7 reports results from Model 2 only.

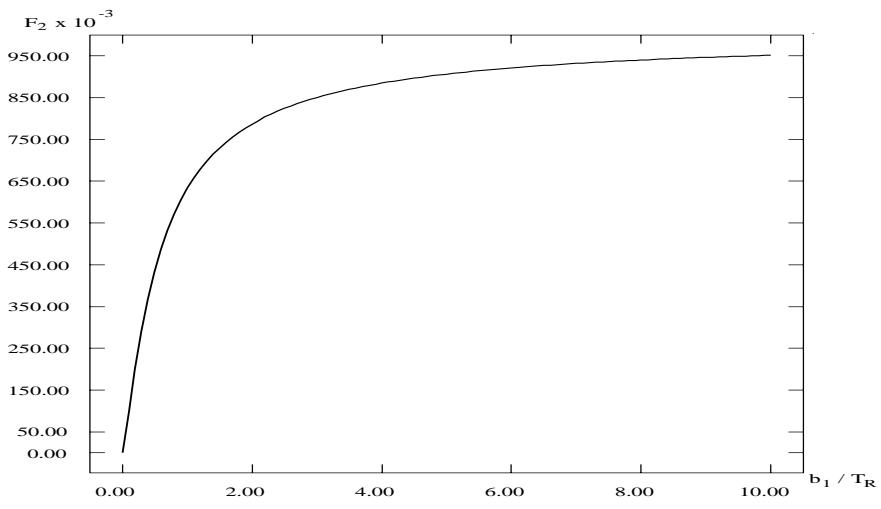

Figure 5: Variation of factor $F_{2}=\frac{b_{1}}{T_{R}}\left(1-e^{\frac{-1}{b_{1} / T_{R}}}\right)$ with respect to $\frac{b_{1}}{T_{R}}$.

\section{Two-Pole Analysis}

The two-pole methodology for interconnect response computation under step input has been discussed in $[4,7,20]$. For interconnect trees (or lines) the transfer function has a special form in which the numerator polynomial is a constant, i.e., approximating to $s^{2}$ term yields $H(s) \approx$ $\frac{1}{1+s b_{1}+s^{2} b_{2}}$. For the case of resistive source $\left(R_{S}\right)$ and capacitive load $\left(C_{L}\right)$ impedances, the transfer function coefficients are given by [8]

$$
\begin{aligned}
& b_{1}=R_{S} C+R_{S} C_{L}+\frac{R C}{2}+R C_{L} \\
& b_{2}=\frac{R_{S} R C^{2}}{6}+\frac{R_{S} R C C_{L}}{2}+\frac{(R C)^{2}}{24}+\frac{R^{2} C C_{L}}{6}+\frac{L C}{2}+L C_{L}
\end{aligned}
$$

For this form of the transfer function, the output response under infinite ramp input is

$$
\begin{aligned}
U_{\text {out }}(s) & =\frac{V_{0}}{T_{R}} \frac{1}{s^{2}} \frac{1}{1+s b_{1}+s^{2} b_{2}} \\
& =\frac{V_{0}}{T_{R}}\left[\frac{-b_{1}}{s}+\frac{1}{s^{2}}-\frac{1+b_{1} s_{2}}{s_{1}-s_{2}} \frac{1}{s-s_{1}}+\frac{1+b_{1} s_{1}}{s_{1}-s_{2}} \frac{1}{s-s_{2}}\right]
\end{aligned}
$$


and the corresponding time-domain response is

$$
u_{\text {out }}(t)=\frac{V_{0}}{T_{R}}\left[-b_{1}+t+\frac{1+b_{1} s_{2}}{s_{2}-s_{1}} e^{s_{1} t}+\frac{1+b_{1} s_{1}}{s_{1}-s_{2}} e^{s_{2} t}\right] U(t)
$$

where $U(t)$ is the unit step function. The time-domain response for a finite ramp is

$$
\begin{aligned}
v_{\text {out }}(t)= & u_{\text {out }}(t)-u_{\text {out }}\left(t-T_{R}\right) \\
= & \frac{V_{0}}{T_{R}}\left[T_{R}+\frac{\left(1+b_{1} s_{2}\right)\left(e^{s_{1} t}-e^{s_{1}\left(t-T_{R}\right)}\right)}{\left(s_{2}-s_{1}\right)}\right. \\
& \left.+\frac{\left(1+b_{1} s_{1}\right)\left(e^{s_{2} t}-e^{s_{2}\left(t-T_{R}\right)}\right)}{\left(s_{1}-s_{2}\right)}\right] U(t)
\end{aligned}
$$

Note that the first and second moments of the transfer function can be obtained from the coefficients $b_{1}$ and $b_{2}$, i.e., $M_{1}=b_{1}$ and $M_{2}=b_{1}^{2}-b_{2}$. We use the coefficient notation $b_{1}, b_{2}$ and the moment notation $M_{1}, M_{2}$ interchangeably according to the simplicity of the expression.

\subsection{Threshold Delay Models}

Depending on the sign of $b_{1}^{2}-4 b_{2}$, the poles of the transfer function can be either real or complex. However, for most cases of interest the poles turn out to be real, and we now discuss delay models for the case of real poles. The condition for the poles to be real is $\left(b_{1}^{2}-4 b_{2}\right)=$ $\left(4 M_{2}-3 M_{1}^{2}\right) \geq 0$. Since the magnitude $\left|s_{2}\right|$ is greater than $\left|s_{1}\right|$, the second term in the time-domain response decreases rapidly compared to the first term. Hence, the two-pole infinite ramp response can be approximated as

$$
u_{\text {out }}(t) \approx \frac{V_{0}}{T_{R}}\left[-b_{1}+t+\frac{1+b_{1} s_{2}}{s_{2}-s_{1}} e^{s_{1} t}\right]
$$

and the finite ramp response as

$$
v_{\text {out }}(t) \approx \frac{V_{0}}{T_{R}}\left[T_{R}+\frac{1+b_{1} s_{2}}{s_{2}-s_{1}}\left(e^{s_{1} t}-e^{s_{1}\left(t-T_{R}\right)}\right)\right] .
$$

Note that the residue $k_{1}=\frac{1+b_{1} s_{2}}{s_{2}-s_{1}}$ is a positive quantity, and that the pole $s_{1}$ has to be negative in value for the response to converge.

\section{Threshold Delay for Infinite Ramp Response.}

Model 4. The delay $T_{R D 4}$ at threshold voltage $u_{t h}$ can be obtained as

$$
T_{R D 4}+\frac{1+b_{1} s_{2}}{s_{2}-s_{1}} e^{s_{1} T_{R D 4}}=u_{t h} T_{R}+b_{1}
$$

Again, we can solve such a recursive equation in less than 10 iterations of simple back-substitution (with $T_{A D}$ as the starting value) for all the interconnect configurations we considered. Another way to evaluate the above iterative equation is by substituting some $f\left(T_{A D}\right)$ for $T_{R D 4}$ in the exponential term, which yields

$$
T_{R D 4}=u_{t h} T_{R}+b_{1}-\frac{1+b_{1} s_{2}}{s_{2}-s_{1}} e^{s_{1} f\left(T_{A D}\right)}
$$

where $f\left(T_{A D}\right)$ depends on the threshold voltage and $T_{A D}$. For example, for $50 \%$ threshold voltage $f\left(T_{A D}\right)=T_{A D}$ and for $90 \%$ threshold voltage $f\left(T_{A D}\right)=2.3 T_{A D}$. We found that the delay values using Equation (16) are close to the values obtained by solving the equation through iteration. Similar to the analysis of Model 1, a better approximation for the $f\left(T_{A D}\right)$ term can be obtained by expressing $T_{R D 4}$ as $u_{t h} T_{R}+\tau_{R D 4}$, since $T_{R D 4}$ is greater than the threshold delay for the infinite ramp input $\left(u_{t h} T_{R}\right)$.

\section{Threshold Delay for Finite Ramp Response.}

Model 5. The delay $T_{R D 5}$ at threshold voltage $v_{t h}$ can be obtained from the response as

$$
v_{t h} T_{R}=T_{R}-\frac{1+b_{1} s_{2}}{s_{2}-s_{1}}\left(e^{-s_{1} T_{R}}-1\right) e^{s_{1} T_{R D 5}} .
$$

Since the value of the pole $s_{1}$ is negative, the quantity $\left(e^{-s_{1} T_{R}}-1\right)$ is positive and the residue $\frac{1+b_{1} s_{2}}{s_{2}-s_{1}}$ is also positive. Thus, the delay expression reduces to

$$
T_{R D 5}=\frac{1}{\left|s_{1}\right|}\left|\ln \left(\frac{F_{3}}{\left(1-v_{t h}\right)}\right)\right|
$$

where the factor $F_{3}=\frac{\left(1+b_{1} s_{2}\right)\left(e^{\left|s_{1}\right| T_{R}}-1\right)}{\left(s_{2}-s_{1}\right) T_{R}}$ can vary widely.

Model 6. Since the threshold delay computed from the finite ramp response is greater than $T_{R}$, an alternative formula for the threshold delay can be obtained by assuming the form $T_{R D 6}=T_{R}+\tau_{R D 6}$. Substituting into Equation (15) yields

$$
v_{t h} T_{R}=T_{R}-\frac{1+b_{1} s_{2}}{s_{2}-s_{1}}\left(1-e^{s_{1} T_{R}}\right) e^{s_{1} \tau_{R D 6}} .
$$

Therefore, the delay is

$$
T_{R D 6}=T_{R}+\frac{1}{\left|s_{1}\right|}\left|\ln \left(\frac{F_{4}}{\left(1-v_{t h}\right)}\right)\right|
$$

where the factor $F_{4}=\frac{\left(1+b_{1} s_{2}\right)\left(1-e^{\left.-\left|s_{1}\right| T_{R}\right)}\right.}{T_{R}\left(s_{2}-s_{1}\right)}$ varies over only a small range. For the range of interconnect configurations studied both Model 5 and Model 6 gave essentially identical results, and hence Section 7 reports results from Model 5 only. [9] gives a detailed discussion of two-pole models for the case of complex poles.

\section{Interconnection Trees}

Finally, we describe how to extend our analytical models to estimate delays in arbitrary interconnect trees. An $R L C$ network is called an $R L C$ tree if it does not contain a closed path of resistors and inductors, i.e., all resistors and inductors are floating with respect to ground, and all capacitors are connected to ground. Consider an $R L C$ interconnect tree with root (or source) $S$ and set of sinks (or leaves) $\{1,2, \ldots, n\}$. The unique path from root $S$ to the sink node $i$ is denoted by $p(i)$ and is referred to as the main path. The edges/nodes not on the main path are referred to as the off-path edges/nodes. We model each edge on the main path of the tree using a lumped $R L C$ segment, e.g., an $\mathbf{L}, \mathbf{T}$, or $\pi$ model. ${ }^{6}$

We approximate the off-path subtree rooted at node $i$ with its admittance. At any node $i$, the admittance $Y_{i}$ is equal to (i) the capacitance of node $i\left(C_{i}\right)$ if there is no subtree at node $i$, or (ii) to the sum of the capacitance of node $i\left(C_{i}\right)$ and the subtree admittance $Y_{T(i)}$ otherwise. In other words,

$$
\begin{array}{lll}
Y_{i} & =s C_{i} & \text { if node } i \text { has no off-path subtree } \\
s C_{i}+Y_{T(i)} & \text { if node } i \text { has an off-path subtree }
\end{array}
$$

With this approximation, the main path reduces to an $R L Y$ equivalent circuit. Only two admittance moments need to be computed for an exact transfer function moment computation for the main path. The $k^{\text {th }}$

\footnotetext{
${ }^{6}$ Our model is not limited to traditional segment models, and accuracy of our results would likely improve if we use non-uniform segment models [7, 19] designed to perfectly match the low-order moments of the distributed $R L C$ line.
} 


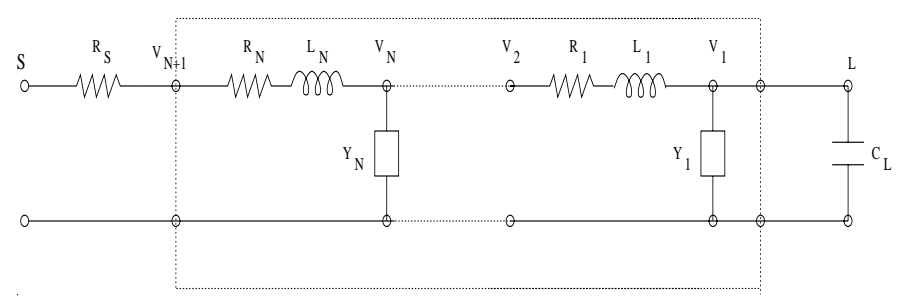

Figure 6: Representation of the main path in the tree, where each distributed line is modeled using $R L C$ segments. $Y_{i}$ indicates the off-path subtree admittance at node $i$.

coefficient $b_{k}$ of the transfer function for the general $R L Y$ circuit of Figure 6 can be obtained using the recursive equation given in [7]. The first and second coefficients of the transfer function are

$$
\begin{aligned}
b_{1}^{S L}= & R_{S} \sum_{j=1}^{N} Y_{1, j}+R_{N} \sum_{j=1}^{N} Y_{1, j}+b_{1}^{N} \\
b_{2}^{S L}= & R_{S} \sum_{j=1}^{N} Y_{1, j} \cdot b_{1}^{j}+R_{S} \sum_{j=1}^{N} Y_{2, j}+R_{N} \sum_{j=1}^{N} Y_{1, j} \cdot b_{1}^{j} \\
& +R_{N} \sum_{j=1}^{N} Y_{2, j}+L_{N} \sum_{j=1}^{N} Y_{1, j}+b_{2}^{N}
\end{aligned}
$$

The first and second moments are expressed in terms of coefficients as $M_{1}=b_{1}$ and $M_{2}=b_{1}^{2}-b_{2}$. For any given source-sink pair the coefficients $b_{1}$ and $b_{2}$ can be computed in linear time by traversing the main path and using Equation (19) to obtain transfer function coefficients.

\section{Experimental Results}

We evaluate the above models by simulating various $R L C$ interconnect lines with different source/load impedances and different input rise times. We consider typical interconnect parameters encountered in single-chip interconnects [8], with the length of the interconnect being $2000 \mu \mathrm{m}$. The source resistance is varied between 100 to $1000 \Omega$ and the load capacitance is varied from 0.1 to $1.0 \mathrm{pf}$. We also consider $100 \mathrm{ps}$ and 500 $p s$ rise times for the input ramp.

For all our experiments, we compute exact $50 \%$ and $90 \%$ delays from the response at the load using the SPICE3e simulator. The step input delay is computed using the Elmore delay formula and then multiplying it with the appropriate constant for the given threshold voltage. For example, Elmore delay at $50 \%$ threshold voltage is $0.69 b_{1}$ and at $90 \%$ threshold voltage is $2.3 b_{1}$. Unlike [5], we find that Elmore delay is not at all close to SPICE-computed 50\% threshold delays and, depending on the rise time of the signal and driver resistance, can be either greater or less than SPICE-computed delays (e.g., when the rise time is 500 ps the Elmore delay is for most cases less than the SPICE-computed delays). Also, increased rise time of the input signal causes the Elmore delay to deviate further from SPICE-computed delays (see Tables 1 and $3)$.

For comparison, we also present delay estimates using the analytical ramp delay model $T_{A D}$. When the rise time of the ramp input is increased from $100 \mathrm{ps}$ to $500 \mathrm{ps}$ the SPICE delays at $50 \%$ threshold are increased by approximately $200 \mathrm{ps}$, which suggests that delay at $50 \%$ threshold voltage is proportional to $\frac{T_{R}}{2}$. This effect of the rise time is well modeled in the analytical ramp delay model $T_{A D}$. To compute ramp input delays using the single-pole methodology we use either the Model 1 or Model 2, depending on the value of the first moment $b_{1}$ and the threshold voltage of interest. Similarly, to compute ramp input delays using the two-pole methodology we use either Model 4 or Model 5, again depending on the value of $b_{1}$ and the threshold voltage of interest. (If the delay is computed using the infinite ramp response then we mark those delays in the Table with $\left({ }^{*}\right)$.) Tables 1 and 2 give $50 \%$ and $90 \%$ delay estimates for ramp input with $100 \mathrm{ps}$ rise time. Tables 3 and 4 give 50\% and $90 \%$ delay estimates for ramp input with $500 \mathrm{ps}$ rise time. Over our range of test cases, Elmore delay estimates can be as much as $100 \%$ away from the SPICE-computed delays. In contrast, our single-pole delay estimates are within $4 \%$ of SPICE delays and the two-pole delay estimates are within $2.3 \%$ of SPICE delays.

\section{Conclusions}

Fast delay estimation methods, as opposed to simulation techniques, are needed for incremental performance-driven layout synthesis. Estimation methods based on Elmore delay for a step input, although efficient, cannot accurately estimate the delay for $R L C$ interconnect lines. We have obtained new analytical delay models under ramp input, based on the first and second moments of $R L C$ interconnection lines. The resulting delay estimates are significantly more accurate than Elmore delay estimates. We also describe how to extend our delay models to estimate source-sink delays in arbitrary interconnect trees.

\section{REFERENCES}

[1] L. N. Dworsky, Modern Transmission Line Theory and Applications, Wiley, 1979.

[2] W. C. Elmore, "The Transient Response of Damped Linear Networks with Particular Regard to Wideband Amplifiers", Journal of Applied Physics 19, Jan. 1948, pp. 55-63.

[3] E. G. Friedman and J. H. Mulligan, Jr, "Ramp Input Response of RC Tree Networks", IEEE ASIC Conference, 1996.

[4] M. A. Horowitz, "Timing Models for MOS Circuits", PhD Thesis, Stanford University, Jan. 1984.

[5] R. Gupta et al., "The Elmore Delay as a Bound for RC Trees with Generalized Input Signals", ACM/IEEE Design Automation Conference, June 1995, pp. 364-369.

[6] C. C. Huang and L. L. Wu, "Signal Degradation Through Module Pins in VLSI Packaging", IBM J. Res. and Dev. 31(4), July 1987, pp. 489-498.

[7] A. B. Kahng and S. Muddu, "Two-pole Analysis of Interconnection Trees", Proc. IEEE MCMC Conf., January 1995, pp. 105-110.

[8] A. B. Kahng and S. Muddu,' "Accurate Analytical Delay Models for VLSI Interconnects”, IEEE Int. Symposium on Circuits and Systems, May 1996.

[9] A. B. Kahng and S. Muddu, "Analytical Delay Model for VLSI Interconnects Under Ramp Input”, UCLA CS Dept. TR-960015, April 1996.

[10] T. Lin and C. A. Mead, "Signal Delay in General RC Networks", IEEE Trans. on Computer-Aided Design, Oct. 1984, pp. 331-349.

[11] S. P. McCormick and J. Allen, “ Waveform Moment Methods for Improved Interconnection Analysis", Proc. 27th ACM/IEEE Design Automation Conf., June 1990, pp. 406-412.

[12] L. T. Pillage and R. A. Rohrer, "Asymptotic Waveform Evaluation for Timing Analysis”, IEEE Trans. on CAD 9, Apr. 1990, pp.352-366.

[13] V. Raghavan, J. E. Bracken and R. A. Rohrer, "AWESpice: A General Tool for the Accurate and Efficient Simulation of Interconnect Problems", Proc. 29th ACM/IEEE Design Automation Conf., June 1992, pp. 87-92.

[14] C. L. Ratzlaff, N. Gopal, and L. T. Pillage, "RICE: Rapid Interconnect Circuit Evaluator", Proc. 28th ACM/IEEE Design Automation Conf., June 1991, pp. 555-560.

[15] J. Rubinstein, P. Penfield and M. A. Horowitz, "Signal Delay in RC Tree Networks", IEEE Trans. on CAD 2(3), July 1983, pp. 202-211.

[16] N. Shirali, "Simple Expressions for Interconnect Delay and Input Transition Time", manuscript, 1995.

[17] M. Sriram and S. M. Kang, "Fast Approximation of The Transient Response of Lossy Transmission Line Trees", Proc. ACM/IEEE Design Automation Conf., June 1993, pp. 691-696.

[18] J. Vlach et al., "Group Delay as an Estimate of Delay in Logic", IEEE Trans. on Computer-Aided Design 10, July 1991,pp. 949-953.

[19] Q. Yu and E. S. Kuh, "Exact Moment Matching Model of Transmission Lines and Application to Interconnect Delay Estimation", IEEE Trans. VLSI Systems 3, June 1995, pp. 311-322.

[20] D. Zhou, S. Su, F. Tsui, D. S. Gao and J. S. Cong,"A Simplified Synthesis of Transmission Lines with A Tree Structure", Intl. Journal of Analog Integrated Circuits and Signal Processing 5, Jan. 1994, pp. 19-30. 


\begin{tabular}{|c|c|c|c|c|c|c|c|}
\hline $\begin{array}{l}\text { Interc. } \\
\text { para. }\end{array}$ & $\begin{array}{l}\text { Driver } \\
\text { Res. }\end{array}$ & $\begin{array}{l}\text { Load } \\
\text { Cap. }\end{array}$ & $\overline{\overline{\text { SPICE }}}$ & $\begin{array}{c}\text { Elmore } \\
\text { Delay }\end{array}$ & $\begin{array}{l}\text { Analy. } \\
\text { Delay }\end{array}$ & $\begin{array}{c}\text { Single } \\
\text { Pole }\end{array}$ & $\begin{array}{l}\text { Two } \\
\text { Pole }\end{array}$ \\
\hline $\mathbf{r}, \mathbf{l}, \mathbf{c}$ & $\overline{\mathbf{R}_{S}}$ & $\mathbf{C}_{\mathbf{T}}$ & & $0.693 b_{1}$ & $\mathbf{T}_{\mathrm{AD}}$ & & \\
\hline$/ \mu m$ & $\Omega$ & $p f$ & $p s$ & ps & $p s$ & $p s$ & ps \\
\hline \multirow[t]{9}{*}{$\begin{array}{l}0.0015 \Omega \\
0.176 f f \\
0.246 p h \\
\end{array}$} & 100 & 0.01 & 83 & 25 & 87 & $83^{*}$ & $84^{*}$ \\
\hline & 500 & 0.01 & 178 & 126 & 232 & 178 & 178 \\
\hline & 1000 & 0.01 & 302 & 251 & 413 & 302 & 303 \\
\hline & 100 & 0.1 & 90 & 32 & 96 & $90^{*}$ & $92^{*}$ \\
\hline & 500 & 0.1 & 209 & 157 & 277 & 209 & 209 \\
\hline & 1000 & 0.1 & 364 & 314 & 503 & 365 & 365 \\
\hline & 100 & 1 & 150 & 96 & 189 & 149 & 151 \\
\hline & 500 & 1 & 522 & 471 & 730 & 522 & 522 \\
\hline & 1000 & 1 & 989 & 939 & 1406 & 990 & 990 \\
\hline \multirow{10}{*}{$\begin{array}{c}0.015 \Omega \\
0.176 \mathrm{ff} \\
0.246 \mathrm{ph}\end{array}$} & & & & & & & \\
\hline & 100 & 0.01 & 87 & 29 & 92 & $87 *$ & $88 *$ \\
\hline & 500 & 0.01 & 181 & 129 & 237 & 182 & 182 \\
\hline & 1000 & 0.01 & 305 & 255 & 418 & 306 & 307 \\
\hline & 100 & 0.1 & 96 & 37 & 103 & $96^{*}$ & $97^{*}$ \\
\hline & 500 & 0.1 & 214 & 162 & 284 & 214 & 215 \\
\hline & 1000 & 0.1 & 369 & 319 & 510 & 370 & 371 \\
\hline & 100 & 1 & 172 & 118 & 220 & 171 & 173 \\
\hline & 500 & 1 & 543 & 493 & 761 & 544 & 545 \\
\hline & 1000 & 1 & 1010 & 961 & 1437 & 1012 & 1013 \\
\hline
\end{tabular}

Table 1: The length of the interconnect line in these experiments is always $h=2000 \mu \mathrm{m}$. The rise time of the input ramp is $100 \mathrm{ps}$. For single-pole delay estimates we use Model 1 or 2 and for two-pole estimates we use Model 4 or 5, depending on whether the delay point falls into the infinite ramp response range or the finite ramp response range. The delay estimates refer to $50 \%$ threshold voltage. $\left({ }^{*}\right)$ indicates that the delay is computed using the infinite ramp response models.

\begin{tabular}{||c|c|c|c|c|c|c|c||}
\hline \hline $\begin{array}{c}\text { Interc. } \\
\text { para. }\end{array}$ & $\begin{array}{c}\text { Driver } \\
\text { Res. }\end{array}$ & $\begin{array}{c}\text { Load } \\
\text { Cap. }\end{array}$ & SPICE & $\begin{array}{c}\text { Elmore } \\
\text { Delay }\end{array}$ & $\begin{array}{c}\text { Analy. } \\
\text { Delay }\end{array}$ & $\begin{array}{c}\text { Single } \\
\text { Pole }\end{array}$ & $\begin{array}{c}\text { Two } \\
\text { Pole }\end{array}$ \\
\hline $\mathbf{r , l , c}$ & $\mathbf{R}_{\mathbf{S}}$ & $\mathbf{C}_{\mathbf{T}}$ & & $\mathbf{0 . 6 9 3 b _ { 1 }}$ & $\mathbf{T}_{\mathbf{A D}}$ & & \\
\hline$/ \mu m$ & $\Omega$ & $p f$ & $p s$ & $p s$ & $p s$ & $p s$ & $p s$ \\
\hline \hline $\begin{array}{c}0.0015 \Omega \\
0.176 f f \\
0.246 p h\end{array}$ & 100 & 0.01 & 287 & 25 & 287 & $287^{*}$ & $287^{*}$ \\
\hline$"$ & 500 & 0.01 & 413 & 126 & 432 & $415^{*}$ & $415^{*}$ \\
\hline$"$ & 1000 & 0.01 & 529 & 251 & 613 & 530 & 530 \\
\hline$"$ & 100 & 0.1 & 296 & 32 & 296 & $296^{*}$ & $296^{*}$ \\
\hline$"$ & 500 & 0.1 & 445 & 157 & 477 & $445^{*}$ & $449^{*}$ \\
\hline$"$ & 1000 & 0.1 & 586 & 314 & 703 & 587 & 587 \\
\hline$"$ & 100 & 1 & 380 & 96 & 389 & $380^{*}$ & $381^{*}$ \\
\hline$"$ & 500 & 1 & 736 & 471 & 930 & 736 & 737 \\
\hline$"$ & 1000 & 1 & 1197 & 939 & 1606 & 1197 & 1198 \\
\hline \hline $0.015 \Omega$ & 100 & 0.01 & 291 & 29 & 292 & $292^{*}$ & $292^{*}$ \\
\hline $\begin{array}{l}176 \\
0.246\end{array}$ & & & & & & & \\
\hline$"$ & 500 & 0.01 & 416 & 129 & 437 & $419^{*}$ & $419^{*}$ \\
\hline$"$ & 1000 & 0.01 & 532 & 255 & 618 & 533 & 534 \\
\hline$"$ & 100 & 0.1 & 303 & 37 & 303 & $303^{*}$ & $303^{*}$ \\
\hline$"$ & 500 & 0.1 & 450 & 162 & 484 & $455^{*}$ & $455^{*}$ \\
\hline$"$ & 1000 & 0.1 & 591 & 319 & 710 & 591 & 592 \\
\hline$"$ & 100 & 1 & 405 & 118 & 420 & $406^{*}$ & $408^{*}$ \\
\hline$"$ & 500 & 1 & 757 & 493 & 961 & 758 & 759 \\
\hline$"$ & 1000 & 1 & 1217 & 961 & 1637 & 1219 & 1221 \\
\hline \hline
\end{tabular}

Table 3: The length of the interconnect line in these experiments is always $h=2000 \mu \mathrm{m}$. The rise time of the input ramp is $500 \mathrm{ps}$. The delay estimates refer to $50 \%$ threshold voltage. $\left({ }^{*}\right)$ indicates that the delay is computed using infinite ramp response models.

\begin{tabular}{||c|c|c|c|c|c|c|c||}
\hline \hline $\begin{array}{c}\text { Interc. } \\
\text { para. }\end{array}$ & $\begin{array}{c}\text { Driver } \\
\text { Res. }\end{array}$ & $\begin{array}{c}\text { Load } \\
\text { Cap. }\end{array}$ & SPICE & $\begin{array}{c}\text { Elmore } \\
\text { Delay }\end{array}$ & $\begin{array}{c}\text { Analy. } \\
\text { Delay }\end{array}$ & $\begin{array}{c}\text { Single } \\
\text { Pole }\end{array}$ & $\begin{array}{c}\text { Two } \\
\text { Pole }\end{array}$ \\
\hline $\mathbf{r}, \mathbf{l}, \mathbf{c}$ & $\mathbf{\mathbf { R } _ { \mathbf { S } }}$ & $\mathbf{C}_{\mathbf{T}}$ & & $\mathbf{0 . 6 9 3 \mathbf { b } _ { \mathbf { 1 } }}$ & $\mathbf{T}_{\mathbf{A D}}$ & & \\
\hline$\mu \mathrm{\mu} m$ & $\Omega$ & $p f$ & $p s$ & $p s$ & $p s$ & $p s$ & $p s$ \\
\hline \hline $\begin{array}{c}0.0015 \Omega \\
0.176 f f \\
0.246 p h\end{array}$ & 100 & 0.01 & 141 & 85 & 87 & 145 & 143 \\
\hline$"$ & 500 & 0.01 & 468 & 418 & 232 & 470 & 469 \\
\hline$"$ & 1000 & 0.01 & 882 & 835 & 413 & 886 & 885 \\
\hline$"$ & 100 & 0.1 & 161 & 106 & 96 & 165 & 161 \\
\hline$"$ & 500 & 0.1 & 572 & 522 & 277 & 574 & 573 \\
\hline$"$ & 1000 & 0.1 & 1090 & 1042 & 503 & 1094 & 1093 \\
\hline$"$ & 100 & 1 & 366 & 319 & 189 & 372 & 366 \\
\hline$"$ & 500 & 1 & 1612 & 1563 & 730 & 1615 & 1614 \\
\hline$"$ & 1000 & 1 & 3167 & 3118 & 1406 & 3172 & 3170 \\
\hline \hline $\begin{array}{l}0.015 \Omega \\
0.176 f f \\
0.246 p h\end{array}$ & 100 & 0.01 & 150 & 96 & 92 & 156 & 151 \\
\hline$"$ & 500 & 0.01 & 476 & 430 & 237 & 482 & 479 \\
\hline$"$ & 1000 & 0.01 & 889 & 846 & 418 & 898 & 895 \\
\hline$"$ & 100 & 0.1 & 174 & 123 & 103 & 181 & 175 \\
\hline$"$ & 500 & 0.1 & 583 & 539 & 284 & 591 & 587 \\
\hline$"$ & 1000 & 0.1 & 1100 & 1059 & 510 & 1111 & 1107 \\
\hline$"$ & 100 & 1 & 429 & 392 & 220 & 445 & 435 \\
\hline$"$ & 500 & 1 & 1668 & 1636 & 761 & 1688 & 1682 \\
\hline$"$ & 1000 & 1 & 3218 & 3191 & 1437 & 3245 & 3238 \\
\hline \hline
\end{tabular}

Table 2: The length of the interconnect line in these experiments is always $h=2000 \mu \mathrm{m}$. The rise time of the input ramp is $100 \mathrm{ps}$. The delay estimates refer to $90 \%$ threshold voltage.

\begin{tabular}{|c|c|c|c|c|c|c|c|}
\hline $\begin{array}{c}\text { Interc. } \\
\text { para. }\end{array}$ & $\begin{array}{c}\begin{array}{c}\text { Driver } \\
\text { Res. }\end{array} \\
\end{array}$ & $\begin{array}{l}\text { Load } \\
\text { Cap. }\end{array}$ & SPICE & $\begin{array}{c}\text { Elmore } \\
\text { Delay }\end{array}$ & $\begin{array}{l}\text { Analy. } \\
\text { Delay }\end{array}$ & $\begin{array}{c}\text { Single } \\
\text { Pole }\end{array}$ & $\begin{array}{l}\text { Two } \\
\text { Pole } \\
\end{array}$ \\
\hline $\mathbf{r}, \mathbf{l}, \mathbf{c}$ & $\overline{\mathbf{R}_{\mathrm{S}}}$ & $\mathbf{C}_{\mathrm{T}}$ & & $0.693 b_{1}$ & $T_{A D}$ & & \\
\hline$/ \mu m$ & $\Omega$ & $p f$ & $p s$ & $p s$ & $p s$ & $p s$ & $p s$ \\
\hline \multirow[t]{9}{*}{$\begin{array}{l}0.0015 \Omega \\
0.176 f f \\
0.246 p h \\
\end{array}$} & 100 & 0.01 & 487 & 85 & 287 & $487 *$ & $487 *$ \\
\hline & 500 & 0.01 & 720 & 418 & 432 & 722 & 721 \\
\hline & 1000 & 0.01 & 1110 & 835 & 613 & 1113 & 1113 \\
\hline & 100 & 0.1 & 496 & 106 & 296 & $496^{*}$ & $496^{*}$ \\
\hline & 500 & 0.1 & 814 & 522 & 477 & 816 & 816 \\
\hline & 1000 & 0.1 & 1312 & 1042 & 703 & 1315 & 1315 \\
\hline & 100 & 1 & 633 & 319 & 389 & 638 & 634 \\
\hline & 500 & 1 & 1826 & 1563 & 930 & 1830 & 1828 \\
\hline & 1000 & 1 & 3374 & 3118 & 1606 & 3379 & 3378 \\
\hline \multirow{10}{*}{$\begin{array}{c}0.015 \Omega \\
0.176 \mathrm{ff} \\
0.246 \mathrm{ph} \\
\end{array}$} & & & & & & & \\
\hline & 100 & 0.01 & 491 & 96 & 292 & $492 *$ & $492 *$ \\
\hline & 500 & 0.01 & 727 & 430 & 437 & 732 & 730 \\
\hline & 1000 & 0.01 & 1116 & 846 & 618 & 1124 & 1122 \\
\hline & 100 & 0.1 & 503 & 123 & 303 & 504 & 504 \\
\hline & 500 & 0.1 & 825 & 539 & 484 & 832 & 833 \\
\hline & 1000 & 0.1 & 1322 & 1059 & 710 & 1332 & 1329 \\
\hline & 100 & 1 & 687 & 392 & 420 & 700 & 692 \\
\hline & 500 & 1 & 1882 & 1636 & 961 & 1902 & 1896 \\
\hline & 1000 & 1 & 3425 & 3191 & 1637 & 3452 & 3446 \\
\hline
\end{tabular}

Table 4: The length of the interconnect line in these experiments is always $h=2000 \mu \mathrm{m}$. The rise time of the input ramp is $500 \mathrm{ps}$. The delay estimates refer to $90 \%$ threshold voltage. 